\title{
Passively Q-Switched Erbium-Doped Fiber Laser Based on GeSe Saturable Absorber
}

\author{
Ying Liu, Zhifeng Hong, Xiaojuan Liu*, Ran Zhang, Shiliang Yin \\ College of Physics and Optoelectronic Engineering, Shandong University of Technology, Zibo, China \\ Email: *liuxiaojuansd@163.com
}

How to cite this paper: Liu, Y., Hong, Z.F., Liu, X.J., Zhang, R. and Yin, S.L. (2021) Passively Q-Switched Erbium-Doped Fiber Laser Based on GeSe Saturable Absorber. Optics and Photonics Journal, 11, 89-103.

https://doi.org/10.4236/opj.2021.114007

Received: April 12, 2021

Accepted: April 23, 2021

Published: April 26, 2021

Copyright $\odot 2021$ by author(s) and Scientific Research Publishing Inc. This work is licensed under the Creative Commons Attribution International License (CC BY 4.0).

http://creativecommons.org/licenses/by/4.0/

\begin{abstract}
GeSe nanosheets were prepared by ultrasonic-assisted liquid phase exfoliation (LPE), and the nonlinear saturated absorption performance was experimentally studied. The modulation depth and saturation intensity of the prepared GeSe saturable absorber (SA) were $15 \%$ and $1.44 \mathrm{MW} / \mathrm{cm}^{2}$, respectively. Using the saturated absorption characteristics of GeSe SA, a passively Q-switched erbium-doped fiber laser was systematically demonstrated. As the pump power increases, the pulse repetition frequency increases from $22.8 \mathrm{kHz}$ to 77.59 $\mathrm{kHz}$. The shortest pulse duration is $1.51 \mu \mathrm{s}$, and the corresponding pulse energy is $46.14 \mathrm{~nJ}$. Experimental results show that GeSe nanosheets can be used as high-efficiency SA in fiber lasers. Our results will provide a useful reference for demonstrating pulsed fiber lasers based on GeSe equipment.
\end{abstract}

\section{Keywords}

GeSe Nanosheets, GeSe Saturable Absorber, Passively Q-Switched Erbium-Doped Fiber Laser

\section{Introduction}

Compared with continuous light (CW) lasers, ultrafast fiber lasers have shorter pulse duration and higher pulse energy, and have been extensively studied. So far, the use of saturable absorbers (SAs) for passive Q-switching and mode-locking is the main technique to modulate CW into a pulse state [1]-[10]. In the process of exploring new SAs, researchers are paying more and more attention to ideal SA materials with high nonlinearity, fast response time, wide absorption band, ambient atmospheric stability, low cost, and low loss [11]-[32]. In recent years, new materials such as quantum dots [33] [34], metal nanoparticles [35] [36] [37] [38], and two-dimensional (2D) materials have gradually entered our field of vision, and have been proven to have excellent saturable absorption characteris- 
tics. Among them, 2D materials have many unique optoelectronic properties that traditional materials do not have, especially their excellent nonlinear optical properties, which show great potential in manufacturing high-performance and new functional optoelectronic devices. In 2004, people successfully produced a 2D material graphene [39], and proved that it has saturable absorption characteristics. Since then, 2D materials such as topological insulators (TI) [40] [41], black phosphorus (BP) [42] [43], MXenes [44] [45], and transition metal dichalcogenides (TMDs) have attracted widespread attention, and most of them have been used as SA in optical operations.

TMDs, as a new type of functional materials for optoelectronic applications, has recently attracted widespread research interest [6]-[12]. TMDs are layered materials with the general formula $\mathrm{MX}_{2}$, where $\mathrm{M}$ is a transition metal $(\mathrm{Nb}, \mathrm{Ta}$, $\mathrm{Ti}, \mathrm{Mo}, \mathrm{W}, \ldots)$ and $\mathrm{X}$ is a chalcogen element $(\mathrm{S}, \mathrm{Se}, \mathrm{Te})$. The layers, made of triangular lattices of transition metal atoms sandwiched by covalently bonded chalcogens, are held together by weak van der Waals forces, which is the reason why TMDs can be readily exfoliated into thin flakes down to the single-layer limit. In addition, when the material changes from a multilayer to a single layer, the TMDs will transition from an indirect band gap to a direct band gap, which indicates that the band gap of TMDs can be designed. This feature brings some excellent optical properties, such as high carrier mobility, controllable photoelectron properties and outstanding nonlinear optical absorption [6] [7] [8]. However, current TMDs materials usually show a large band gap in visible light or near-infrared light, so the absorption at mid-infrared wavelengths is relatively weak. In recent years, the layered TMDs nanostructures composed of IV-VI elements such as $\mathrm{GeS}, \mathrm{GeSe}, \mathrm{GeSe}_{2}$, and SnSe have attracted great interest. Their band gap is usually in the range of $0.5-1.5 \mathrm{eV}$. GeSe, GeS and other germanium-based layered nanostructures are alternatives to traditional TMDs such as $\mathrm{MoS}_{2}$ and $\mathrm{WS}_{2}$. They are expected to become SAs for fiber lasers due to their relatively high stability and environmental sustainability. Among the materials with the M-X structure, GeSe is the only material with a direct band gap, and the direct and indirect band gaps are closely placed. It belongs to a narrow band gap p-type semiconductor, and its band gap is in the range of $1.1-1.2 \mathrm{eV}$ [46]. These characteristics make it possible to use SAs as fiber lasers. GeSe crystals are composed of vertically stacked layers, which are held together by weaker van der Waals force. Atoms are connected by covalent bonds with a strong force, which can eliminate dangling bonds and surface states. In addition, GeSe has a highly anisotropic crystal with a layered structure, where the layers are parallel to the growth direction. Because the surface of the GeSe nanosheet is chemically inert, it guarantees its high chemical and environmental stability. In 2013, B. Mukherjee et al. proved that photodetectors based on GeSe nanosheets have parameters comparable to other two-dimensional materials [47].

In this contribution, we used ultrasonic-assisted liquid phase exfoliation (LPE) to prepare GeSe nanosheets. The modulation depth and saturation intensity of GeSe SA prepared were $15 \%$ and $1.44 \mathrm{MW} / \mathrm{cm}^{2}$, respectively. In addition, we 
demonstrated a passively Q-switched erbium-doped fiber laser operating at $1560.4 \mathrm{~nm}$ and designed a simple ring cavity based on GeSe SA. As the pump power increases, the pulse repetition frequency increases from $22.8 \mathrm{kHz}$ to 77.59 $\mathrm{kHz}$. The shortest pulse duration is $1.51 \mu \mathrm{s}$, and the corresponding pulse energy is $46.14 \mathrm{~nJ}$. Experimental results show that GeSe nanosheets can be used as high-efficiency SA in fiber lasers. Our results will provide a useful reference for demonstrating pulsed fiber lasers based on GeSe equipment.

\section{Preparation and Characterization of GeSe SA}

In this work, an ultrasonic-assisted LPE method was used as a simple and low-cost method to prepare GeSe nanosheets. Mix $60 \mathrm{mg}$ of GeSe powder with $60 \mathrm{ml}$ of pure water, and ultrasonically treat at a low temperature for 10 hours to obtain GeSe dispersion. The dispersion was then centrifuged in a high-speed centrifugal device at $3000 \mathrm{rpm}$ for 20 minutes until the GeSe powder was fully dispersed. Finally, we select the upper $80 \%$ solution, as shown in Figure 1, for subsequent experiments.

First, the GeSe-PVA film is manufactured. The preparation process is shown in Figure 2, $400 \mathrm{mg}$ of polyvinyl acetate (PVA) powder was added to $10 \mathrm{ml}$ of GeSe solution, stirred for one hour to fully dissolve the PVA, and the mixture was sonicated for 1 hour to prepare a uniform GeSe-PVA dispersion. The experiment uses condensed PVA, which is a white powdery resin that can be dissolved in water at room temperature and can support GeSe materials well. At the same time, PVA has a good model, the film made is not easy to tear and wear-resistant, and has good permeability in the visible light to infrared light wave range. Therefore, it is easy to form a thin film on the end face of the optical

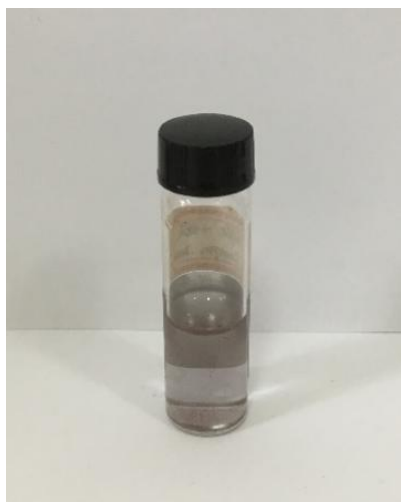

Figure 1. Supernatant liquor of the GeSe nanosheets.

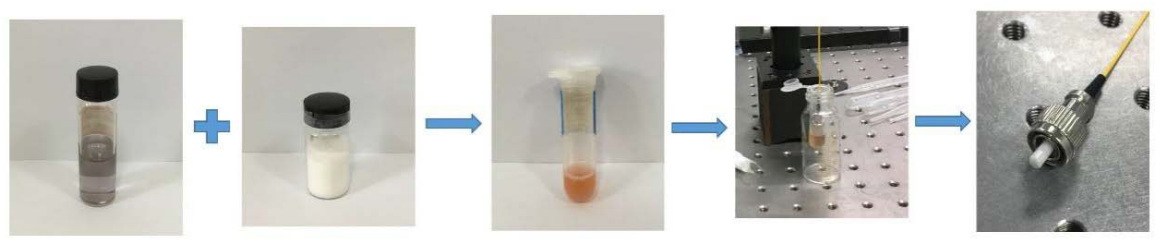

Figure 2. Preparation process diagram of GeSe SA. 
fiber connector without affecting the properties of the material. Then, in order to manufacture GeSe SA, the end face of the optical fiber connector was immersed in the supernatant for 20 minutes. Finally, the optical fiber connector soaked with the material is dried at room temperature for 24 hours, thereby forming a GeSe-PVA film on the end face of the optical fiber connector. Connect it to a clean optical fiber connector with a flange, so that GeSe SA is completed.

As shown in Figure 3(a) and Figure 3(b), the clear appearance and uniform lattice edges of GeSe were analyzed by scanning electron microscope (SEM) and transmission electron microscopy (TEM). In order to determine the specific phase of the GeSe sample, the high-resolution transmission electron microscopy (HRTEM) is shown in Figure 3(b). It's seen that there is no obvious defect, and the lattice distance is about $0.36 \mathrm{~nm}$ corresponding to the $(2,0,1)$ lattice plane.

We measured the Raman spectrum of GeSe nanosheets at an excitation wavelength of $514 \mathrm{~nm}$, as shown in Figure 4. It can be seen from the figure that GeSe nanosheets have two characteristic peaks, namely the $\mathrm{B}_{2 \mathrm{u}}$ peak and the $\mathrm{A}_{\mathrm{g}}$ peak, and their Raman frequency shifts correspond to $149.8 \mathrm{~cm}^{-1}$ and $188.7 \mathrm{~cm}^{-1}$, respectively. This result corresponds to the reported Raman peak of GeSe, which can well prove that the prepared material is GeSe [48] [49].

In addition, in order to confirm the thickness of the prepared GeSe-PVA film, we measured it with an atomic force microscope (AFM). Figure 5(a) is an AFM image of a GeSe-PVA film. We arbitrarily select two different positions and label them as A and B respectively. From the height profile in Figure 5(b), we can see that the thickness of the GeSe-PVA film prepared in the study area is about 5 nm.

The balanced twin detector measurement scheme is used to measure the transmission of samples at different power to study the nonlinear saturated absorption characteristics of GeSe SA at $1550 \mathrm{~nm}$. A fiber laser with central wavelength of $1550 \mathrm{~nm}$, pulse width of $6 \mathrm{~ns}$ and repetition rate of $10 \mathrm{~Hz}$ is used. The intensity dependent nonlinear normalized saturation absorption curve of the GeSe-PVA film is shown in the blue point in Figure 6. Note that with the increasing of light intensity, the nonlinear optical transmission increases to about
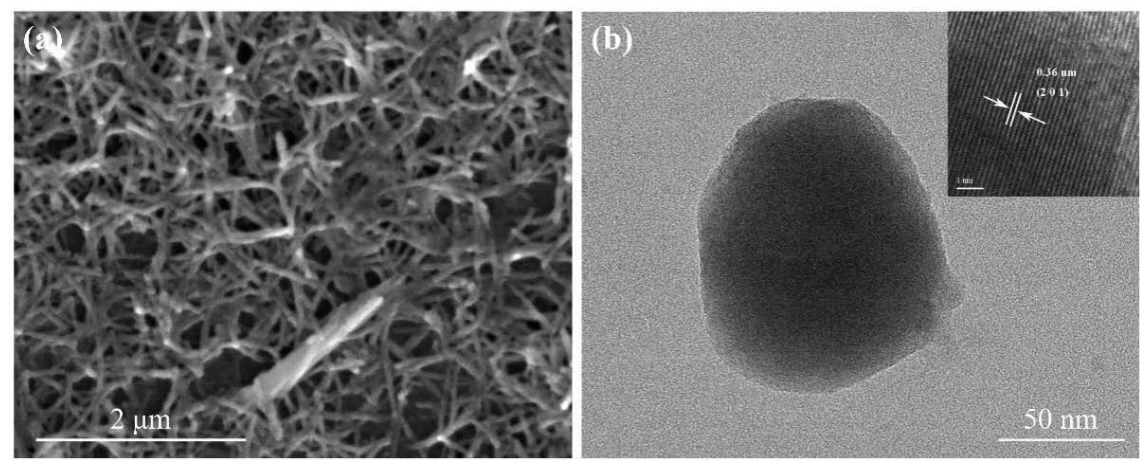

Figure 3. (a) SEM image of GeSe nanosheets; (b) TEM image of GeSe nanosheets. The inset is HRTEM of GeSe nanosheets image. 


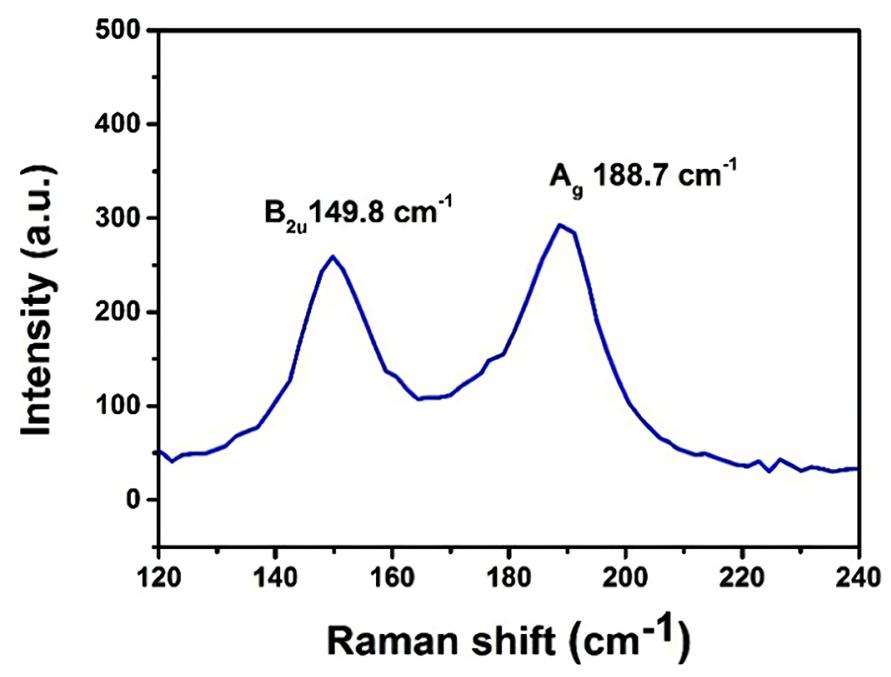

Figure 4. Raman spectrum of GeSe nanosheets.

(a)

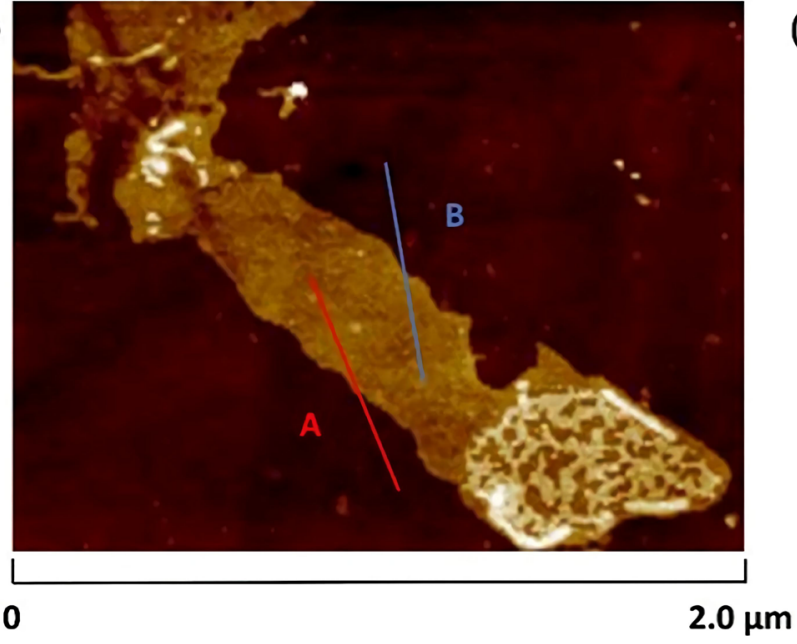

(b)

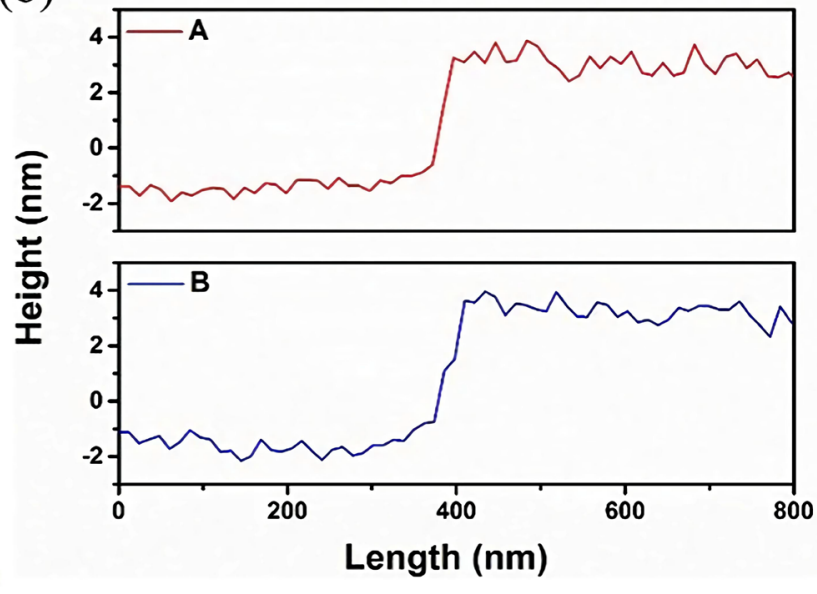

Figure 5. (a) The AFM of the GeSe-PVA film; (b) The height profile of GeSe-PVA film.

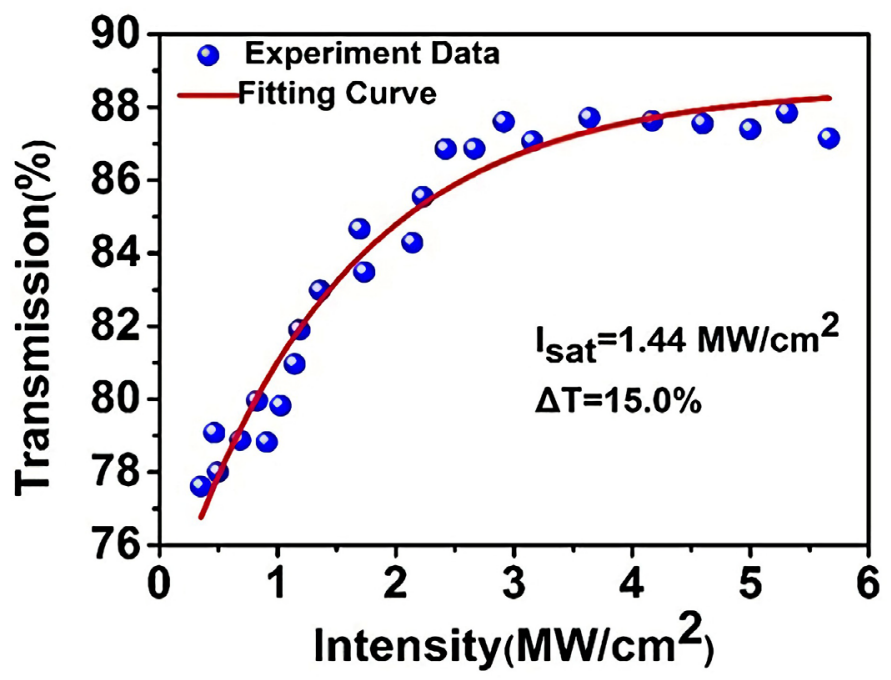

Figure 6. Saturable absorption curve of GeSe SA. 
$87 \%$, and there is a saturation, which shows a typical saturated absorption characteristic. Through fitting analysis, as shown in the red line in Figure 6, the data are very consistent with the curve of saturated absorption formula:

$$
T(I)=1-\Delta T \times \exp \left(-I / I_{\text {sat }}\right)-T_{\text {ns }}
$$

where $T(I)$ is transmission, $\Delta T$ is the modulation depth, $I$ is input laser intensity, $I_{\text {sat }}$ and $T_{\text {ns }}$ are saturation intensity and nonsaturable absorbance, respectively. According to the fitting analysis, the modulation depth and saturation intensity of the prepared GeSe-PVA film are provided to be $15 \%$ and $1.44 \mathrm{MW} / \mathrm{cm}^{2}$, respectively.

Using UV/VIS/NIR spectrophotometer (U-3500, Hitachi, Japan) was measured linear GeSe nanosheet transmission spectrum in the range of $200-2000$ $\mathrm{nm}$. As shown in Figure 7, the blue line represents the transmittance of a blank quartz substrate; the red line represents the transmittance of GeSe nanosheets on the same substrate. Obviously, in the wavelength range of $400-2000 \mathrm{~nm}$, the transmittance of the GeSe nanosheet and the blank quartz substrate are $91.5 \% \pm$ $0.5 \%$ and $92.8 \% \pm 0.5 \%$, respectively. The net transmittance of GeSe nanosheets is about $98.7 \%$, and the scattering loss is $2.3 \%$. The results further show that the prepared GeSe nanosheet is an excellent absorber, showing high absorption from ultraviolet (UV) to near-infrared (NIR). GeSe SA has a very wide range of applications.

A good SA should have a high modulation depth ( $10 \%$ for fiber lasers) and a low saturation intensity value. As mentioned above, GeSe SA has a relatively large modulation depth of $15 \%$ and a lower saturation intensity of $1.44 \mathrm{MW} / \mathrm{cm}^{2}$, which is more suitable for achieving nonlinear saturation absorption of ultrafast pulses.

\section{Experiment and Results}

Figure 8 is a schematic of a passively Q-switched erbium-doped fiber laser based on GeSe SA. The experimental device is a ring cavity structure. A $976 \mathrm{~nm}$ laser

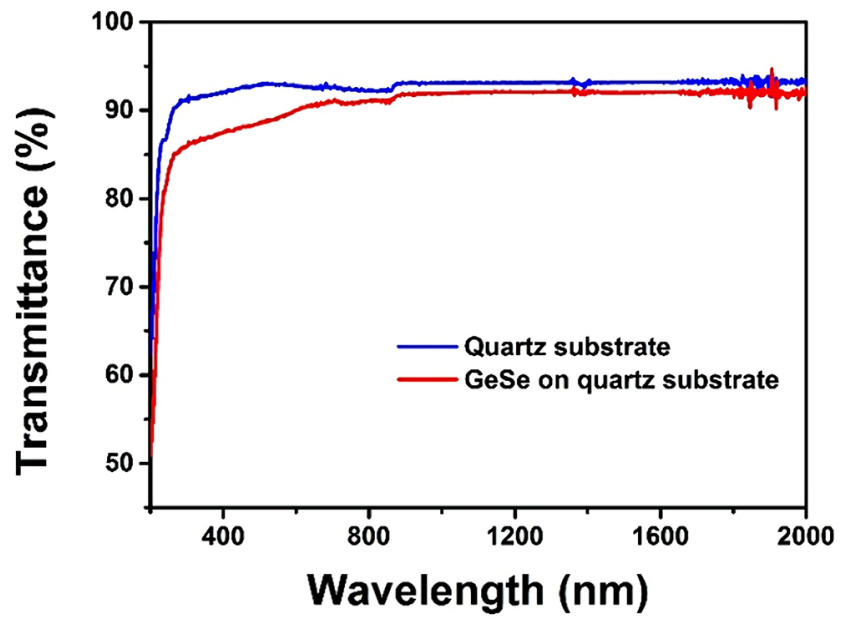

Figure 7. Linear transmission spectra of GeSe nanosheets. 


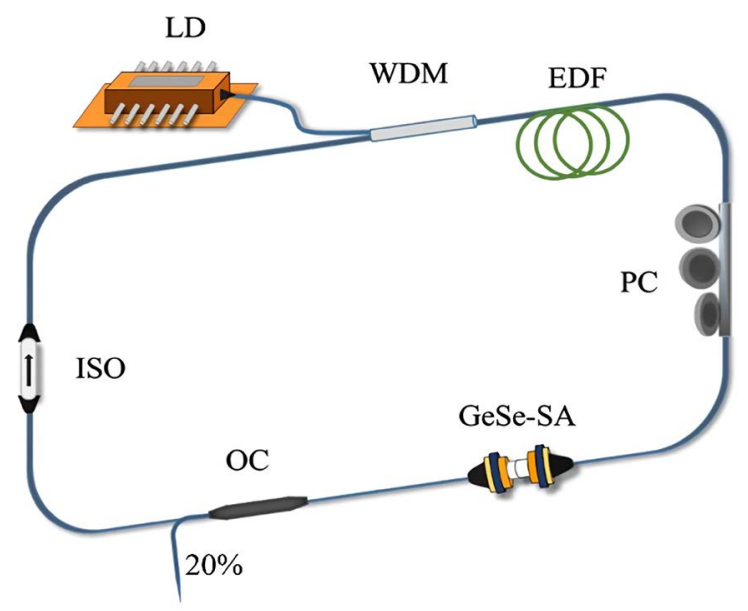

Figure 8. Experimental setup of Q-switched erbium-doped fiber laser based on GeSe SA.

diode (LD) with a maximum pump power of $515 \mathrm{~mW}$ was used as the pump source, which is coupled into the laser cavity through a 980/1550 nm wavelength-division multiplexing (WDM). Use $6 \mathrm{~m}$ long EDF (Nufern-EDFC-980-HP) as the gain medium. The prepared GeSe SA is used as a Q-switched device. A polarization independent isolator (PI-ISO) is used to ensure unidirectional operation of signal inside the cavity. In order to optimize the laser output, a polarization controller (PC) is utilized to adjust the polarization state of circulating light. In addition, a fiber output coupler (OC) with an output ratio of 20:80 is added to the cavity, in which $20 \%$ of the laser is used for output. The total cavity length is about $11 \mathrm{~m}$. The output spectrum, pulse sequence and average output power are respectively detected by a spectrum analyzer (YOKOGAWA AQ6370B), a digital oscilloscope (DP04104), and a power meter (Molectron PM3).

During the experiment, we increased the pump power while using a spectrometer and an oscilloscope to observe the output characteristics of the pulse in the laser. When the pump power was increased to $25 \mathrm{~mW}$, we observed the continuous wave output in the laser in the oscilloscope. Then, when the pump power reached $47 \mathrm{~mW}$, Q-switched pulse output began to appear in the laser. Throughout the experiment, as the pump power changes, the intensity of each pulse in the corresponding pulse sequence has been kept within a similar range, indicating that the output pulse has good stability. When the pump power is increased to the maximum value of $515 \mathrm{~mW}$, the Q-switched pulse output is still stable. Figure 9 shows the pulse output characteristics when the pump power is 515 $\mathrm{mW}$. Figure $9(\mathrm{a})$ is a single pulse sequence diagram. From the figure, we can see that there are some spikes at the top of the pulse, which causes the pulse envelope to be not smooth enough, but no jitter is found in the oscilloscope. The shortest pulse duration when the pump power is $515 \mathrm{~mW}$ is $1.51 \mu \mathrm{s}$. The illustration shows the output pulse sequence at this time, the intensity of each pulse is basically the same, and the repetition frequency at this time can be obtained as $77.59 \mathrm{kHz}$. Figure 9(b) is the spectrum of the output pulse. The center wavelength corresponds to $1560.4 \mathrm{~nm}$. 
(a)

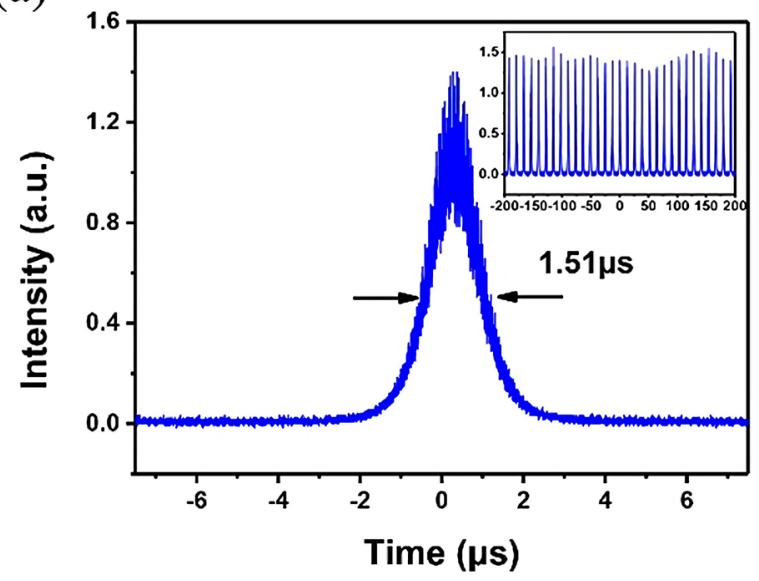

(b)

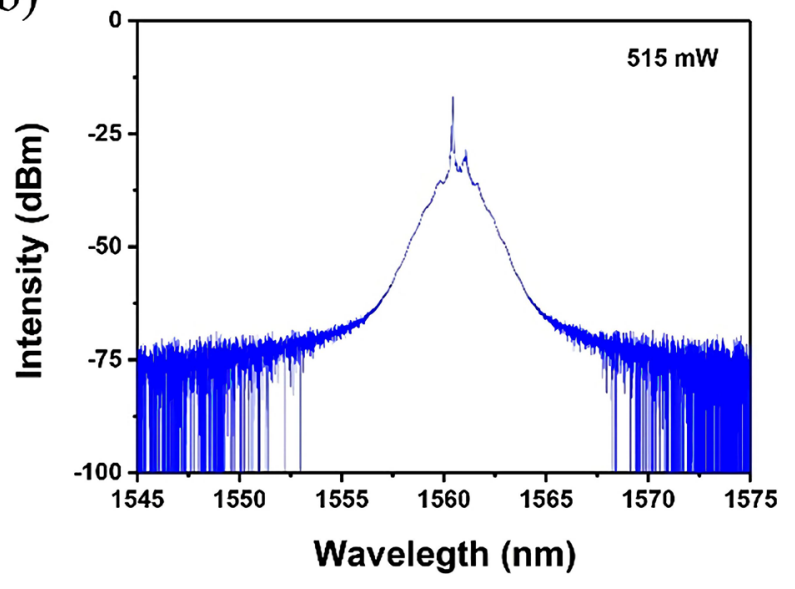

Figure 9. Output pulse at $515 \mathrm{~mW}$ pump power. (a) Single pulse, the inset is pulse sequence; (b) Pulse spectrum.

When the pump power increases from $47 \mathrm{~mW}$ to $515 \mathrm{~mW}$, the pulse duration and repetition frequency change with the pump power as shown in Figure 10(a). It can be seen from the figure that when the pump power is $47 \mathrm{~mW}$, the pulse duration is $13.85 \mu \mathrm{s}$, and the corresponding repetition frequency is 22.8 $\mathrm{kHz}$. When the pump power is $515 \mathrm{~mW}$, the pulse duration is reduced to $1.51 \mu \mathrm{s}$, and the repetition frequency is increased to $77.59 \mathrm{kHz}$. Obviously, the pump power is inversely proportional to the pulse duration and directly proportional to the repetition frequency. Figure 10(b) depicts the relationship between the output power and the single pulse energy with the pump power. When the pump power is $515 \mathrm{~mW}$, the maximum average output power obtained is $3.58 \mathrm{~mW}$, and the maximum single pulse energy is $46.14 \mathrm{~nJ}$. It can be calculated that the light-light efficiency at this time is $0.7 \%$, and the slope efficiency is $0.75 \%$. In the experiment, the slope efficiency is relatively low. There are many reasons for this result, such as GeSe SA parameters, cavity design, OC split ratio and pump power limitation. Therefore, we can further optimize the parameters of the cavity and GeSe SA, and at the same time replace the OC, pump source and other experimental devices to improve the slope efficiency. Limited by the pump power in the experiment, the damage threshold of GeSe SA could not be obtained. Therefore, we infer that the use of a pump source with a larger pump power can achieve a higher repetition rate and further shorten the pulse duration, so that a better performance output pulse can be obtained.

Throughout the experiment, the fiber laser can obtain stable Q-switched pulses in the pump power range of $47-515 \mathrm{~mW}$. Figure 11 shows the pulse sequence at different pump powers. The entire experimental process lasts for one month, during which the Q-switched pulse sequence remains highly stable. Facts have proved that the GeSe nanosheet film is not damaged under the current pump power range, and under normal environmental conditions, it has a high degree of stability and oxidation resistance. This plays a vital role in the practical application of optical devices. 

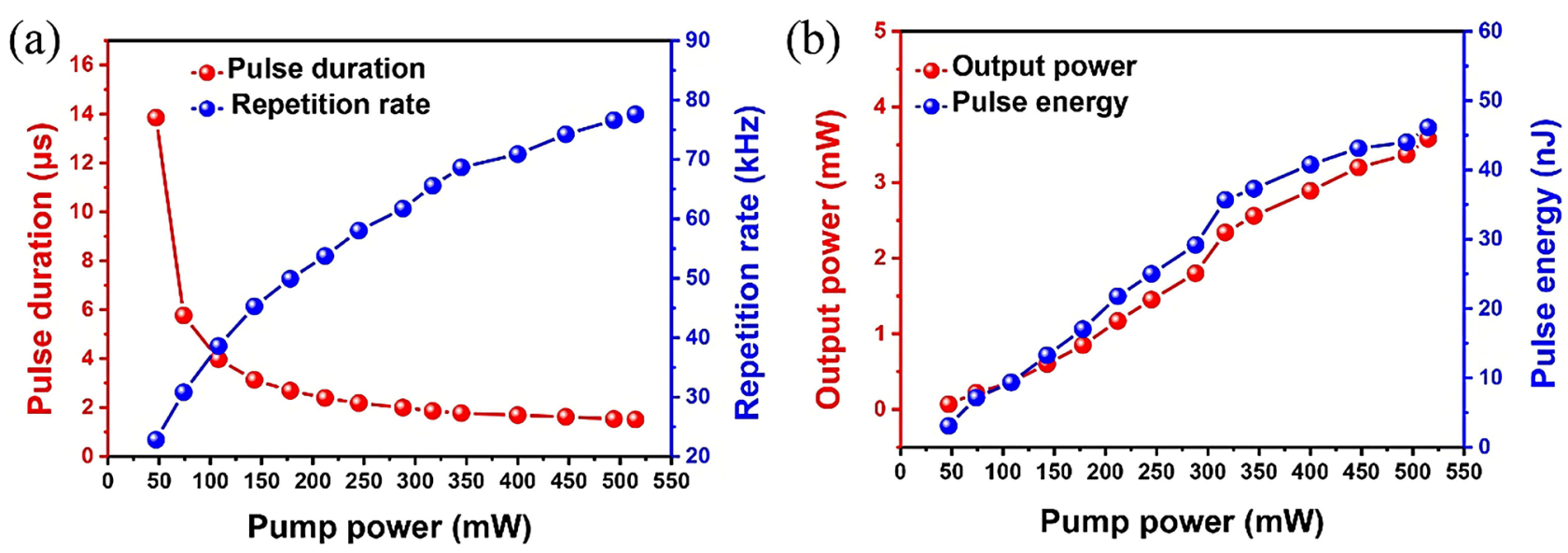

Figure 10. (a) The pulse duration and pulse repetition rate versus the pump power; (b) The output power and pulse energy versus the pump power.

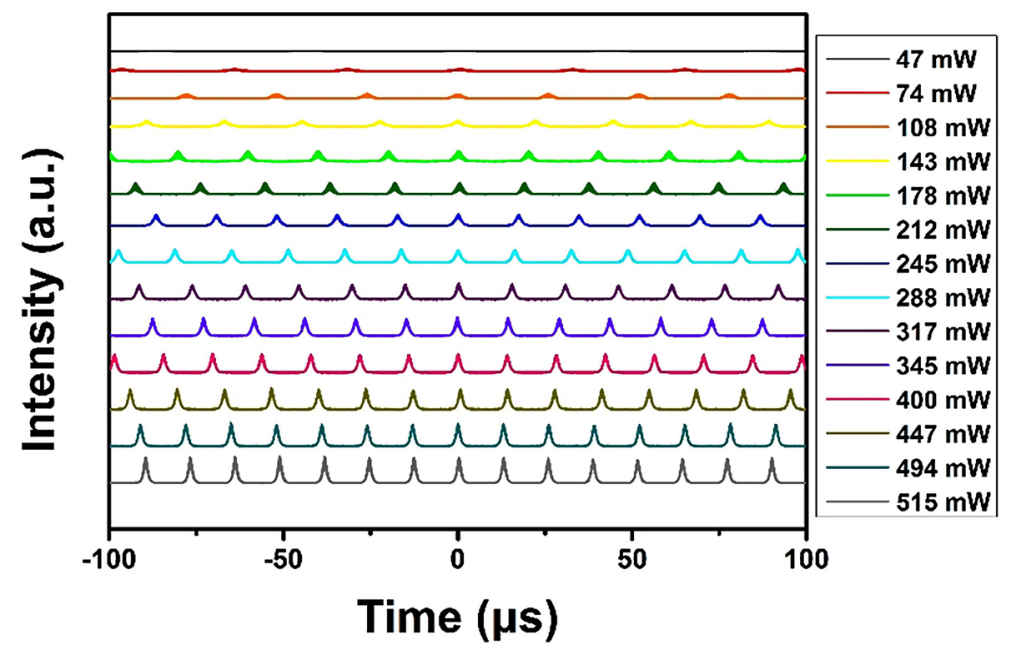

Figure 11. Pulse sequences at different powers.

In order to test the long-term stability of the pulse output, we have performed multiple measurements on the spectrum at a pump power of $515 \mathrm{~mW}$. Recorded every 0.5 -hour interval, a total of five sets of data are recorded, as shown in Figure 12.

When the pump power is too high, the Q-switched operation will often disappear due to the pulse saturation. However, in our experiments, GeSe-based SA showed a higher damage threshold and good thermal stability until the pump power was increased to $515 \mathrm{~mW}$. In order to verify the influence of GeSe SA on the erbium-doped fiber laser, we removed the fiber connector with GeSe-PVA film and replaced it with a clean fiber connector of the same length and model. Adjusting the PC while changing the pump power, no matter how the adjustment is made, there is no Q-switched output pulse. Facts have proved that the Q-switched operation is realized in the fiber laser due to GeSe SA. Further comparison shows that GeSe nanosheets can be used as an excellent saturable absorber to achieve Q-switched pulse output. In the experiment, no optical damage 


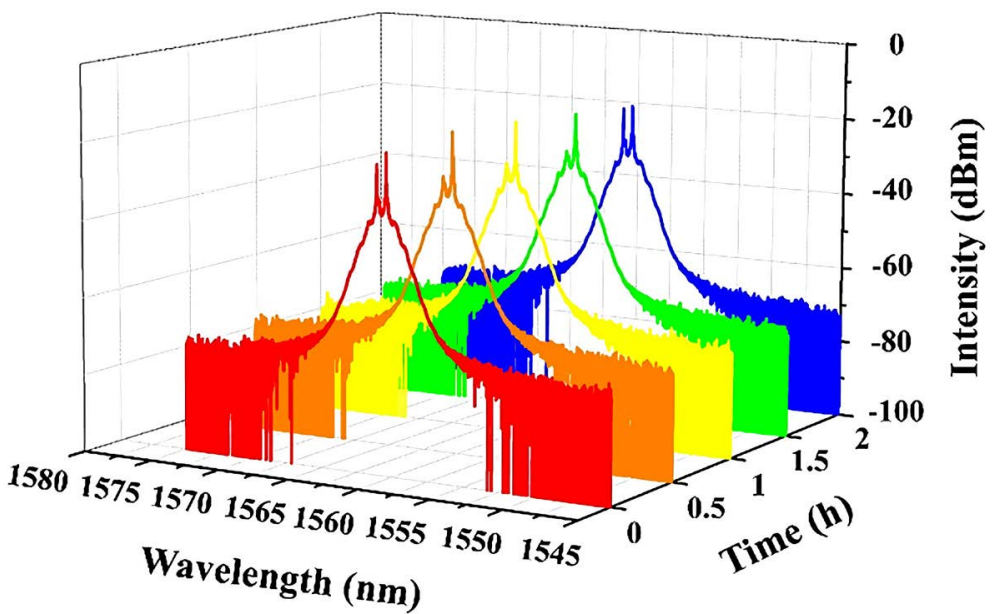

Figure 12. Long-term performance of the Q-switched fiber laser in 2 hours with an interval of 30 minutes.

Table 1. Comparison of passively Q-switched EDFL performance based on different 2D materials.

\begin{tabular}{cccccccc}
\hline Meterials & $\begin{array}{c}\text { Pump } \\
\text { Power }(\mathrm{mW})\end{array}$ & $\begin{array}{c}\text { Modulation } \\
\text { Depth }(\%)\end{array}$ & $\begin{array}{c}\text { Repetition } \\
\text { Rate }(\mathrm{KHz})\end{array}$ & $\begin{array}{c}\text { Pulse } \\
\text { durations } \\
(\mu \mathrm{s})\end{array}$ & $\begin{array}{c}\text { Pulse } \\
\text { Energy } \\
(\mathrm{nJ})\end{array}$ & $\begin{array}{c}\text { Output } \\
\text { Power } \\
(\mathrm{mW})\end{array}$ & Refs \\
\hline Graphene & $6.5-82.8$ & 45 & $3.3-65.9$ & 3.7 & 16.7 & 1.1 & {$[50]$} \\
$\mathrm{ZrS}_{2}$ & $55-345$ & 14.7 & $40.65-87.1$ & 1.49 & 33.5 & 2.89 & {$[31]$} \\
$\mathrm{TiS}_{2}$ & $20-39$ & 8.3 & $25.2-50.7$ & 4 & 9.5 & $/$ & {$[51]$} \\
$\mathrm{Bi}_{2} \mathrm{Se}_{3}$ & $96-360$ & 4.3 & $459-940$ & 1.9 & 23.8 & 22.35 & {$[52]$} \\
$\mathrm{BP}^{2}$ & $/$ & 19.5 & $4.43-18$ & 9.35 & 28.3 & $/$ & {$[53]$} \\
$\mathrm{Antimonene}$ & $41-345$ & 13.06 & $25.3-76.7$ & 1.58 & 37.9 & 2.85 & {$[13]$} \\
$\mathrm{Fe}_{3} \mathrm{O}_{4}$ & $15-110$ & 8.2 & $7.8-33.3$ & 3.2 & 23.76 & 0.794 & {$[54]$} \\
$\mathrm{GeSe}^{2}$ & $47-515$ & 15 & $22.8-77.59$ & 1.51 & 46.14 & 3.58 & Our Work \\
\hline
\end{tabular}

occurred in GeSe SA, which indicates that the prepared GeSe SA can withstand higher optical power density.

In order to find out the advantages and disadvantages of passive Q-switched operation based on GeSe SA; Table 1 shows the performance comparison of passive Q-switched erbium-doped fiber lasers based on various 2D materials. The results show that, compared with the previous experimental materials, GeSe SA prepared by the LPE method has a higher modulation depth. In addition, the laser's repetition frequency, pulse duration, pulse energy and average output power also have obvious advantages.

\section{Conclusion}

In a word, this chapter proposes and proves the saturable absorption characteristics of GeSe nanosheets, using GeSe SA to achieve stable Q-switched pulse output in erbium-doped fiber lasers. In our experiments, the modulation depth 
and saturation intensity of the prepared GeSe nanosheets are 15\% and 1.44 $\mathrm{MW} / \mathrm{cm}^{2}$, respectively. A ring cavity in the $1550 \mathrm{~nm}$ band was built, and the Q-switching operation was realized within the range of pump power from 47 $\mathrm{mW}$ to $515 \mathrm{~mW}$, and the repetition frequency was increased from $22.8 \mathrm{kHz}$ to $77.59 \mathrm{kHz}$. Among them, when the pump power is $515 \mathrm{~mW}$, the maximum pulse output power of $3.58 \mathrm{~mW}$ is obtained, the shortest pulse duration is $1.51 \mu \mathrm{s}$, and the corresponding pulse energy is $46.14 \mathrm{~nJ}$. Experimental results show that GeSe nanosheets can be used as high-efficiency SA in fiber lasers. During the whole experiment, the output of the system remained stable. Our results will provide useful references for demonstrating pulsed fiber lasers based on GeSe devices.

\section{Conflicts of Interest}

The authors declare no conflicts of interest regarding the publication of this paper.

\section{References}

[1] Li, L., Lv, R., Liu, S., Chen, Z., Wang, J., Wang, Y., Ren, W. and Liu, W. (2019) Ferroferric-Oxide Nanoparticle Based Q-Switcher for a $1 \mu \mathrm{m}$ Region. Optical Materials Express, 9, 731-738. https://doi.org/10.1364/OME.9.000731

[2] Guo, S.Y., Zhang, Y.P., Ge, Y.Q., Zhang, S.L., Zeng, H.B. and Zhang, H. (2019) 2D V-V Binary Materials: Status and Challenges. Advanced Materials, 31, Article ID: 1902352. https://doi.org/10.1002/adma.201902352

[3] Guo, B., Xiao, Q.L., Wang, S.H. and Zhang, H. (2019) 2D Layered Materials: Synthesis, Nonlinear Optical Properties, and Device Applications. Laser \& Photonics Reviews, 13, Article ID: 1800327. https://doi.org/10.1002/lpor.201800327

[4] Liu, W.J., Liu, M.L., Chen, X., Shen, T., Lei, M., Guo, J.G., Deng, H.X., Zhang, W., Dai, C.Q., Zhang, X.F. and Wei, Z.Y. (2020) Ultrafast Photonics of Two Dimensional $\mathrm{AuTe}_{2} \mathrm{Se}_{4 / 3}$ in Fiber Lasers. Communications Physics, 3, 15. https://doi.org/10.1038/s42005-020-0283-9

[5] Guo, P.L., Li, X.H., Chai, T., Feng, T.C. and Ge, Y.Q. (2019) Few-Layer Bismuthene for Robust Ultrafast Photonics in C-Band Optical Communications. Nanotechnology, 30, Article ID: 354002. https://doi.org/10.1088/1361-6528/ab2150

[6] Niu, K., Chen, Q., Sun, R., Man, B. and Zhang, H. (2017) Passively Q-Switched Erbium-Doped Fiber Laser Based on $\mathrm{SnS}_{2}$ Saturable Absorber. Optical Materials EXpress, 7, 3934-3943. https://doi.org/10.1364/OME.7.003934

[7] Sadeq, S.A., Harun, S.W. and Al-Janabi, A.H. (2018) Ultrashort Pulse Generation with an Erbium-Doped Fiber Laser Ring Cavity Based on a Copper Oxide Saturable Absorber. Applied Optics, 57, 5180-5185. https://doi.org/10.1364/AO.57.005180

[8] Mao, D., She, X.Y., Du, B.B., Yang, D.X., Zhang, W.D., Song, K., Cui, X.Q., Jiang, B.Q., Peng, T. and Zhao, J.L. (2016) Erbium-Doped Fiber Laser Passively Mode Locked with Few-Layer WSe ${ }_{2} / \mathrm{MoSe}_{2}$ Nanosheets. Scientific Reports, 6, Article No. 23583. https://doi.org/10.1038/srep23583

[9] Chen, B.H., Zhang, X.Y., Wu, K., Wang, H. and Chen, J.P. (2015) Q-Switched Fiber Laser Based on Transition Metal Dichalcogenides $\mathrm{MoS}_{2}, \mathrm{MoSe}_{2}, \mathrm{WS}_{2}$, and $\mathrm{WSe}_{2}$. Optics Express, 23, 26723-26737. https://doi.org/10.1364/OE.23.026723

[10] Yan, P.G., Chen, H., Yin, J.D., Xu, Z.H., Li, J.R., Jiang, Z.K., Zhang, W.F., Wang, 
J.Z., Li, I.L., Sun, Z.P. and Ruan, S.C. (2017) Large-Area Tungsten Disulfide for Ultrafast Photonics. Nanoscale, 9, 1871-1877. https://doi.org/10.1039/C6NR09183K

[11] Liu, W.J., Pang, L.H., Han, H.N., Bi, K., Lei, M. and Wei, Z.Y. (2017) Tungsten Disulphide for Ultrashort Pulse Generation in All-Fiber Lasers. Nanoscale, 9, 5806-5811. https://doi.org/10.1039/C7NR00971B

[12] Salman, A.A. and Al-Janabi, A.H. (2019) Q-Switching Near-Infrared Multiwavelength Generation by Intracavity Tungsten-Oxide-Induced Four-Wave Mixing in an Erbium-Doped Fiber Laser. Applied Optics, 58, 4332-4336. https://doi.org/10.1364/AO.58.004332

[13] Hu, P., Liu, Y., Guo, L.P., Ge, X.L., Liu, X.J., Yu, L.J. and Liu, Q.H. (2019) Passively Q-Switched Erbium-Doped Fiber Laser Based on Antimonene as Saturable Absorber. Applied Optics, 58, 7845-7850. https://doi.org/10.1364/AO.58.007845

[14] Luo, Z.Q., Wu, D.D., Xu, B., Xu, H.Y., Cai, Z.P., Peng, J., Weng, J., Xu, S., Zhu, C.H., Wang, F.Q., Sun, Z.P. and Zhang, H. (2016) Two-Dimensional Material-Based Saturable Absorbers: Towards Compact Visible-Wavelength All-Fiber Pulsed Lasers. Nanoscale, 8, 1066-1072. https://doi.org/10.1039/C5NR06981E

[15] Nie, H.K., Sun, X.L., Zhang, B.T., Yan, B.Z., Li, G.R., Wang, Y.R., Liu, J.T., Shi, B.N., Liu, S.D. and He, J.L. (2018) Few-Layer $\mathrm{TiSe}_{2}$ as a Saturable Absorber for Nanosecond Pulse Generation in $2.95 \mu \mathrm{m}$ Bulk Laser. Optics Letters, 43, 3349-3352. https://doi.org/10.1364/OL.43.003349

[16] Yang, W., Xu, N. and Zhang, H. (2018) Nonlinear Absorption Properties of Indium Selenide and Its Application for Demonstrating Pulsed Er-Doped Fiber Laser. Laser Physics Letters, 15, Article ID: 105101. https://doi.org/10.1088/1612-202X/aad896

[17] Song, Y.F., Chen, S., Zhang, Q., Li, L., Zhao, L.M., Zhang, H. and Tang, D.Y. (2016) Vector Soliton Fiber Laser Passively Mode Locked by Few Layer Black Phosphorus-Based Optical Saturable Absorber. Optics Express, 24, 25933-25942. https://doi.org/10.1364/OE.24.025933

[18] Chen, Y., Jiang, G., Chen, S., Guo, Z., Yu, X., Zhao, C., Zhang, H., Bao, Q., Wen, S., Tang, D. and Fan, D. (2015) Mechanically Exfoliated Black Phosphorus as a New Saturable Absorber for Both Q-Switching and Mode-Locking Laser Operation. $O p$ tics Express, 23, 12823-12833. https://doi.org/10.1364/OE.23.012823

[19] Song, Y.F., Liang, Z.M., Jiang, X.T., Chen, Y.X., Jun, Z., Lu, L., Ge, Y.Q., Wang, K., Zheng, J.L., Lu, S.B., Ji, J.H. and Zhang, H. (2017) Few-Layer Antimonene Decorated Microfiber: Ultra-Short Pulse Generation and All-Optical Thresholding with Enhanced Long Term Stability. 2D Materials, 4, Article ID: 045010. https://doi.org/10.1088/2053-1583/aa87c1

[20] Liu, W.J., Liu, M.L., Liu, X.M., Lei, M. and Wei, Z.Y. (2020) SnSSe as a Saturable Absorber for an Ultrafast Laser with Superior Stability. Optics Letters, 45, 419-422. https://doi.org/10.1364/OL.380183

[21] Zhang, Y., Li, X.H., Qyyum, A., Feng, T.C., Guo, P.L., Jiang, J. and Zheng, H.R. (2018) PbS Nanoparticles for Ultrashort Pulse Generation in Optical Communication Region. Particle \& Particle Systems Characterization, 35, Article ID: 1800341. https://doi.org/10.1002/ppsc.201800341

[22] Li, L., Pang, L.H., Zhao, Q.Y., Liu, W.J. and Su, Y.L. (2020) $\mathrm{VSe}_{2}$ Nanosheets for Ultrafast Fiber Lasers. Journal of Materials Chemistry C, 8, 1104-1109. https://doi.org/10.1039/C9TC06159B

[23] Ma, P., Li, J., Zhang, H. and Yang, Z. (2020) Preparation of High-Damage Threshold WS2 Modulator and Its Application for Generating High-Power Large-Energy Bright-Dark Solitons. Infrared Physics and Technology, 105, Article ID: 103257. 
https://doi.org/10.1016/j.infrared.2020.103257

[24] Feng, J.J., Li, X.H., Shi, Z.J., Zhen, C., Li, X.W., Leng, D.Y., Wang, Y.M., Liu, J. and Zhu, L.J. (2020) 2D Ductile Transition Metal Chalcogenides (TMCs): A Novel High-Performance $\mathrm{Ag}_{2} \mathrm{~S}$ Nanosheets for Ultrafast Photonics. Advanced Optical Materials, 8, Article ID: 1901762. https://doi.org/10.1002/adom.201901762

[25] Wang, Q., Kalantar-Zadeh, K., Kis, A., Coleman, J.N. and Strano, M.S. (2012) Electronics and Optoelectronics of Two-Dimensional Transition Metal Dichalcogenides. Nature Nanotechnology, 7, 699-712. https://doi.org/10.1038/nnano.2012.193

[26] Kanazawa, T., Amemiya, T., Ishikawa, A., Upadhyaya, V., Tsuruta, K., Tanaka, T. and Miyamoto, Y. (2016) Few-Layer HfS 2 Transistors. Scientific Reports, 6, Article No. 22277. https://doi.org/10.1038/srep22277

[27] Huang, Y., Luo, Z., Li, Y., Zhong, M., Xu, B., Che, K., Xu, H., Cai, Z., Peng, J. and Weng, J. (2014) Widely-Tunable, Passively Q-Switched Erbium-Doped Fiber Laser with Few-Layer $\mathrm{MoS}_{2}$ Saturable Absorber. Optics Express, 22, 25258-25266. https://doi.org/10.1364/OE.22.025258

[28] Salman, A.A. and Al-Janabi, A.H. (2019) Multiwavelength Q-Switched Erbium-Doped Fibre Laser-Based Aluminum Nanoparticles Saturable Absorber and Sagnac Loop Filter. Laser Physics, 29, Article ID: 065103. https://doi.org/10.1088/1555-6611/ab13b5

[29] Rosdin, R.Z.R.R., Ahmad, F., Ali, N.M., Harun, S.W. and Arof, H. (2014) Q-Switched Er-Doped Fiber Laser with Low Pumping Threshold Using Graphene Saturable Absorber. Chinese Optics Letters, 12, 49-53. https://doi.org/10.3788/COL201412.091404

[30] Kang, Z., Liu, M., Li, Z., Li, S., Jia, Z., Liu, C., Qin, W. and Qin, G. (2018) Passively Q-Switched Erbium Doped Fiber Laser Using a Gold Nanostars Based Saturable Absorber. Photonics Research, 6, 549. https://doi.org/10.1364/PRJ.6.000549

[31] Hu, P., Huang, Y., Liu, F.F., Liu, Y., Guo, L.P., Ge, X.L. and Liu, X.J. (2019) Q-Switched Erbium-Doped Fiber Laser Based on $\mathrm{ZrS}_{2}$ as a Saturable Absorber. Chinese Optics Letters, 17, Article ID: 080603. https://doi.org/10.3788/COL201917.080603

[32] Liu, X.J., Hu, P., Liu, Y., Guo, L.P., Ge, X.L. and Zhang, H.N. (2020) Conventional Solitons and Bound-State Solitons in an Erbium-Doped Fiber Laser Mode-Locked by $\mathrm{TiSe}_{2}$-Based Saturable Absorber. Nanotechnology, 31, Article ID: 365202. https://doi.org/10.1088/1361-6528/ab8fe6

[33] Wei, K.H., Fan, S.H., Chen, Q.G. and Lai, X.M. (2017) Passively Mode-Locked Yb Fiber Laser with PbSe Colloidal Quantum Dots as Saturable Absorber. Optics EXpress, 25, 24901-24906. https://doi.org/10.1364/OE.25.024901

[34] Ming, N., Tao, S.N., Yang, W.Q., Chen, Q.Y., Sun, R.Y., Wang, C., Wang, S.Y., Man, B.Y. and Zhang, H.N. (2018) Mode-Locked Er-Doped Fiber Laser Based on PbS/CdS Core/Shell Quantum Dots as Saturable Absorber. Optics Express, 26, 9017-9026. https://doi.org/10.1364/OE.26.009017

[35] Zhang, H.N. and Liu, J. (2016) Gold Nanobipyramids as Saturable Absorbers for Passively Q-Switched Laser Generation in the $1.1 \mu \mathrm{m}$ Region. Optics Letters, 41, 1150-1152. https://doi.org/10.1364/OL.41.001150

[36] Kang, Z., Xu, Y., Zhang, L., Jia, Z.X., Liu, L., Zhao, D., Feng, Y., Qin, G.S. and Qin, W.P. (2013) Passively Mode-Locking Induced by Gold Nanorods in Erbium-Doped Fiber Lasers. Applied Physics Letters, 103, Article ID: 041105. https://doi.org/10.1063/1.4816516

[37] Zhang, H.N., Li, B.W. and Liu, J. (2017) Gold Nanobipyramids as a Saturable Ab- 
sorber for Passively Q-Switched Yb-Doped Fiber Laser Operation at $1.06 \mu \mathrm{m}$. Laser Physics Letters, 14, Article ID: 025104. https://doi.org/10.1088/1612-202X/aa538d

[38] Kang, Z., Gao, X.J., Zhang, L., Feng, Y., Qin, G.S. and Qin, W.P. (2015) Passively Mode-Locked Fiber Lasers at 1039 and $1560 \mathrm{~nm}$ Based on a Common Gold Nanorod Saturable Absorber. Optical Materials Express, 5, 794-801. https://doi.org/10.1364/OME.5.000794

[39] Novoselov, K.S., et al. (2005) Two-Dimensional Atomic Crystals. Proceedings of the National Academy of Sciences of the United States of America, 102, 10451-10453.

[40] Luo, Z.C., Liu, M., Liu, H., Zheng, X.W., Luo, A.P., Zhao, C.J., Zhang, H., Wen, S.C. and Xu, W.C. (2013) 2 GHz Passively Harmonic Mode-Locked Fiber Laser by a Microfiber-Based Topological Insulator Saturable Absorber. Optics Letters, 38, 5212 5215. https://doi.org/10.1364/OL.38.005212

[41] Xu, N.N., Zhang, H.N. and Man, B.Y. (2018) Various Large-Energy Soliton Operations within an Er-Doped Fiber Laser with Bismuth Selenide as a Saturable Absorber. Applied Optics, 57, 8811-8818. https://doi.org/10.1364/AO.57.008811

[42] Li, J.F., et al. (2016) Black Phosphorus: A Two-Dimension Saturable Absorption Material for Mid-Infrared Q-Switched and Mode-Locked Fiber Lasers. Scientific reports, 6, Article No. 30361. https://doi.org/10.1038/srep30361

[43] Luo, Z.C., Liu, M., Guo, Z.N., Jiang, X.F., Luo, A.P., Zhao, C.J., Yu, X.F., Xu, W.C. and Zhang, H. (2015) Microfiber-Based Few-Layer Black Phosphorus Saturable Absorber for Ultra-Fast Fiber Laser. Optics Express, 23, 20030-20039. https://doi.org/10.1364/OE.23.020030

[44] Jiang, X.T., Liu, S.X., Liang, W.Y., Luo, S.J., He, Z.L., Ge, Y.Q., Wang, H.D., Cao, R., Zhang, F., Wen, Q., Li, J.Q., Bao, Q.L., Fan, D.Y. and Zhang, H. (2018) Broadband Nonlinear Photonics in Few-Layer MXene $\mathrm{Ti}_{3} \mathrm{C}_{2} \mathrm{~T}_{x}(\mathrm{~T}=\mathrm{F}, \mathrm{O}$, or $\mathrm{OH})$. Laser \& Photonics Reviews, 12, Article ID: 1700229. https://doi.org/10.1002/lpor.201700229

[45] Xu, N.N., Ma, P.F., Fu, S.G., Shang, X.X., Jiang, S.Z., Wang, S.Y., Li, D.W. and Zhang, H.N. (2020) Tellurene-Based Saturable Absorber to Demonstrate Large-Energy Dissipative Soliton and Noise-Like Pulse Generations. Nanophotonics, 9, 2783-2795. https://doi.org/10.1515/nanoph-2019-0545

[46] Vaughn Ii, D.D., Patel, R.J., Hickner, M.A., et al. (2010) Single-Crystal Colloidal Nanosheets of GeS and GeSe. Journal of the American Chemical Society, 132, 15170-15172. https://doi.org/10.1021/ja107520b

[47] Mukherjee, B., Cai, Y., Tan, H.R., et al. (2013) NIR Schottky Photodetectors Based on Individual Single-Crystalline GeSe Nanosheet. ACS Applied Materials \& Interfaces, 5, 9594-9604. https://doi.org/10.1021/am402550s

[48] Fukunaga, T., Sugai, S., Kinosada, T., et al. (1981) Observation of New Raman Lines in GeSe and SnSe at Low Temperatures. Solid State Communications, 38, 1049-1052. https://doi.org/10.1016/0038-1098(81)90015-6

[49] Chandrasekhar, H.R. and Zwick, U. (1976) Raman Scattering and Infrared Reflectivity in GeSe. Solid State Communications, 18, 1509-1513.

https://doi.org/10.1016/0038-1098(76)90381-1

[50] Luo, Z., Zhou, M., Weng, J., Huang, G., Xu, H., Ye, C. and Cai, Z. (2010) Graphene-Based Passively Q-Switched Dual-Wavelength Erbium-Doped Fiber Laser. Optics Letters, 35, 3709-3711. https://doi.org/10.1364/OL.35.003709

[51] Zhu, X., Chen, S., Zhang, M., Chen, L., Wu, Q., Zhao, J., Jiang, Q., Zheng, Z. and Zhang, H. (2018) $\mathrm{TiS}_{2}$-Based Saturable Absorber for Ultrafast Fiber Lasers. Photonics Research, 6, C44-C48. https://doi.org/10.1364/PRJ.6.000C44 
[52] Yu, Z., Song, Y., Tian, J., Dou, Z., Guoyu, H., Li, K., Li, H. and Zhang, X. (2014) High-Repetition-Rate Q-Switched Fiber Laser with High Quality Topological Insulator $\mathrm{Bi}_{2} \mathrm{Se}_{3}$ Film. Optics Express, 22, 11508-11515.

https://doi.org/10.1364/OE.22.011508

[53] Jiang, T., Yin, K., Zheng, X., Yu, H. and Cheng, X.A. (2015) Black Phosphorus as a New Broadband Saturable Absorber for Infrared Passively Q-Switched Fiber Lasers.

[54] Bai, X.K., Mou, C.B., Xu, L.X., Wang, T.Y., Pu, S.L., Huang, S.J. and Zeng, X.L. (2016) Passively Q-Switched Erbium-Doped Fiber Laser Using $\mathrm{Fe}_{3} \mathrm{O}_{4}$-Nanoparticle Saturable Absorber. Applied Physics Express, 9, Article ID: 042701.

https://doi.org/10.7567/APEX.9.042701 